\title{
ПРОГНОЗ ГЕОЛОГИЧЕСКОГО РАЗРЕЗА НА ОСНОВЕ ДЕТАЛЬНОЙ ОБРАБОТКИ СЕЙСМИЧЕСКИХ МАТЕРИАЛОВ В ПРОГРАММНО-АЛГОРИТМИЧЕСКОМ КОМПЛЕКСЕ ॥ГЕОСЕЙФ»
}

\author{
Кочегуров Александр Иванович', \\ kaicc@tpu.ru \\ Иванченков Виктор Павлович ${ }^{1}$, \\ am@am.tpu.ru \\ Орлов Олег Викторович', \\ orloff.oleg@mail.ru
Национальный исследовательский Томский политехнический университет, Россия, 634050, г. Томск, пр. Ленина, 30.

Актуальность. Хорошо известно, что основными целями прогнозирования геологического разреза являются: определение вещественного состава осадконакопления, выделение продуктивных толщ и оценки их нефтегазоносности. Эти цели достигаются в том числе на основе детальной обработки и интерпретации сейсморазведочных данных с привязкой их к результатам геофизического исследования скважин. В современных программно-алгоритмических комплексах обработки и интерпретации сейсмической информации для прогноза геологического разреза в качестве диагностических признаков в основном применяются амплитудные и энергетические характеристики отраженных волн. Фазочастотные характеристики для этих целей прогноза практически не используются. Все это приводит к тому, что при решении задач прогноза геологического разреза процент ошибочных решений достаточно велик и выделение ложных аномалий динамических и кинематических параметров отражений встречается достаточно часто.

Цель: на основе детальной обработки сейсмических материалов в программно-алгоритмическом комплексе «Геосейф» получить прогноз геологического разреза по новым информативным параметрам, извлекаемым из мгновенных фазовых спектров отраженных сейсмических волн.

Методы и средства исследования: цифровая обработка сигналов и пространственно-временных полей, дискретное преобразование Фурье, методы математического моделирования, вычислительный эксперимент.

Результаты. Подробно рассмотрен состав программно-алгоритмического комплекса «Геосейф» и перечень решаемых на нем задач. Показано, что общий подход к решению задач на комплексе «Геосейф» связан с формированием выбранного маршрута в соответствии с принятым графом обработки от имеющихся исходных данных к нужным результатам. Приведена структура детальной обработки сейсмических материалов при реализации алгоритмов фазочастотной деконволюции с целью прогноза геологического разреза. В результате применения рассмотренной методики проведен фазовременной анализ территории Двуреченско-Карандашовской зоны и построена карта районирования по выделенным типам геологического разреза. При этом выявленные корреляционные связи значений фазочастотной характеристики с зональными и локальными литологическими и тектоническими неоднородностями разреза интерполируются и экстраполируются в межскважинное пространство.

\section{Ключевые слова}

Прогноз геологического разреза, фазочастотная деконволюция, фазовременный анализ, детальная обработка, программно-алгоритмический комплекс.

\section{Введение}

Одна из основных особенностей современного этапа нефтегазопоисковых работ в Томской области состоит в необходимости более активного вовлечения в разведочный процесс слабо исследованных территорий. К таковым, в полной мере, относится правобережная часть реки Оби, заключающая в себе большой объем потенциально нефтегазоносных юрских, а в зонах наиболее глубокого прогибания, возможно, и триассовых осадочных отложений. Полученные к настоящему времени материалы геологоразведочных работ не дают оснований для полной и однозначной оценки перспектив нефтегазоносности этого комплекса. Кроме того, даже в районах промышленного освоения месторождений, и в пределах самих месторождений, исключительно актуальной остается проблема определения пространственной модели природного резервуара, характеризующегося, как правило, контрастной латеральной изменчивостью фильт- рационно-емкостных свойств. Решение этой проблемы не в последнюю очередь связано с развитием новых, более эффективных методов решения задач прогнозирования геологического разреза [1-4]. Поэтому потребности создания новых, более эффективных методов решения задач прогнозирования геологического разреза (ПГР) с привлечением наиболее информативных параметров регистрируемых сейсмических волн определяют методологический аспект постановки задачи [5-8].

Исходя из решаемых задач при прогнозе, все методы ПГР можно разделить условно на два класса: 1) методы изучения структурных особенностей геологического разреза для более детального расчленения слоистых осадочных толщ; 2) методы определения петрофизических характеристик осадочных толщ, выделение на их основе коллекторских особенностей отложений и оценки их нефтегазоносности.

В настоящее время для детального расчленения слоистых толщ широко применяются методы де- 
конволюции [9-12]. Эти методы становятся весьма актуальными при поиске месторождений нефти и газа в тонкослоистых геологических средах. В этих средах волны, отраженные от границ раздела геологических сред, интерферируют между собой и выделить границы их раздела становится практически невозможно. Кроме того, интерференционная картина осложняется наложением нерегулярных помех. Поэтому для отождествления границ в тонкослоистых средах, как правило, применяются алгоритмы сжатия сигналов, которые основаны на методах деконволюции. Однако в традиционных методах деконволюции фазовые спектры не оцениваются, их принимают либо минимально фазовыми, либо нуль-фазовыми [13-20]. В то же время именно в сложный закон поведения фазовых спектров сейсмических волн и заложена информация о местоположении отражающих границ, а следовательно, разрешающая способность алгоритмов деконволюции во многом связана с корректным использованием информации о фазе сигнала. Кроме того, фазовые спектры содержат информацию о характере напластования, акустических свойствах и толщине слоев, а также неоднородности поглощения геологических сред. Таким образом, характерное поведение фазовых спектров может служить эффективными признаками обнаружения углеводородов (УВ). Поэтому разработка, исследование и применение методов фазочастотной деконволюции при ПГР имеет важное значение и является весьма актуальной задачей.

Однако практическое применение фазочастотных характеристик (ФЧХ) отраженных сейсмических волн для прогноза геологического разреза встречает определенные трудности. Это, в частности, связано с недостаточной изученностью фазовых спектров отраженных волн, их взаимосвязи с конфигурацией границ и параметрами геологического разреза, а также особенностями их изменения в области залежей нефти и газа. Кроме того, необходимо совершенствование существующих и создание новых фазочастотных алгоритмов обработки сейсмической информации, а также изучение возможности привлечения наряду с ФЧХ других динамических характеристик отраженных волн для комплексного решения задач ПГР.

\section{Детализация волнового поля на основе методов деконволюции}

В настоящее время при анализе волновых сейсмических полей, как уже отмечалось выше, широко применяются методы деконволюции. Они позволяют существенно повысить разрешенность сейсмических записей, что в свою очередь дает возможность увидеть мелкие детали (нарушение осей синфазности, прогибы, поднятия и т. п.), не видимые на исходных разрезах. В работе [21] подробно рассмотрены вопросы построения алгоритмов фазочастотной деконволюции сейсмических волн и приведены первые результаты прослеживания волн на моделях и реальных данных. Поэтому, не повторяя рассуждений рассмотренных ранее, приведем только математические соотношения и пояснения к ним, необходимые для понимания структуры и содержания программно-алгоритмического комплекса «Геосейф». Сразу отметим, что алгоритмы фазочастотной деконволюции могут применяться как для оценки времени прихода целевых волн (алгоритмы фазочастотного прослеживания), так и для детального расчленения слоистых неоднородных осадочных толщ и определения формы геологических объектов (алгоритмы фазовременного анализа).

Для решения первой задачи в [21] предложен алгоритм, в основе которого лежат численные расчеты мгновенных фазовых спектров и построение на их основе функции правдоподобия. При этом вычисления спектров осуществляются путем дискретного преобразования Фурье участка сейсмотрассы в окне анализа, которое перемещается по трассе с шагом, равным шагу дискретизации сейсмической трассы. Сформированная функция правдоподобия позволяет оценить временное положение сейсмических сигналов [21] и имеет следующий вид:

$$
L(\tau)=\sum_{k=1}^{n} W\left(f_{k}\right) \cos \left(\varphi\left(f_{k}\right)-2 \pi f_{k} \tau\right),
$$

где $\varphi\left(f_{k}\right)$ - фазовый спектр в текущем окне; $W\left(f_{k}\right)-$ частотная весовая функция.

Выбор частотной весовой функции определяет вид алгоритма обработки: равновесная (единичная весовая функция) и неравновесная (весовая функция может задаваться искусственным образом, например, быть треугольной функцией).

В основу решения второй задачи были положены выделенные ранее характерные особенности поведения фазовых спектров в зависимости от изменения акустических, поглощающих и дисперсионных свойств геологических сред [6]. На основе выделенных особенностей фазовых спектров были сформированы диагностические признаки, которые далее использовались в качестве информативных прогнозных параметров фазовременного анализа (ФАН-анализа) сейсмических полей [8].

Как ранее было показано [8], ФАН-анализ является инструментом построения картины волнового поля, на которой удается выделить даже самые слабые амплитудные отражения. Высокая разрешающая способность ФАН-анализа дает возможность получать изображения, отличные по структуре рисунка и интенсивности колебаний, что позволяет сформировать различные типы разреза на исследуемой площади. Сама процедура анализа достаточно проста [8] и заключается в построении набора функций вида (1) для каждой сейсмической трассы в исследуемой области:

$$
L_{i}(\tau)=\sum_{k=0}^{n-1} W_{i}\left(f_{k}\right) \cos \left(\varphi\left(f_{k}\right)-2 \pi f_{k} \tau\right),
$$

где $i$ - номер синтезированного фильтра $(i=1, \ldots, l)$; $l$ - число фильтров, используемых при обработке. 
На основе сформированных функций (2) строятся ФАН-образы для всех анализируемых трасс, которые в результате их объединения вдоль профиля образуют ФАН-разрез. В качестве эталонных ФАН-образов принимаются образы на пикетах наблюдения, соответствующих положению скважин. На этом этапе используются данные геофизического исследования скважин (ГИС). Для отнесения полученных типов геологического разреза к тому или иному классу используются процедуры автоматической классификации, в основу которых положены нейросетевые технологии распознавания образов.

Как видно из вышеизложенного, для прогнозирования нефтегазоносности продуктивных комплексов используются методы фазочастотной деконволюции, которые базируются на принципиально новых информационных признаках сейсмического волнового поля, заложенных в фазочастотные характеристики отраженных волн. Это потребовало создания нового программно-алгоритмического комплекса, получившего название «Геосейф» .

\section{Описание программно-алгоритмического комплекса «Геосейф»}

Комплекс «Геосейф» предназначен для детальной обработки и интерпретации сейсмических данных, получаемых при поиске месторождений нефти и газа. Исходными данными для него являются временные разрезы общей глубинной точки (ОГТ), а также данные ГИС.

Перечень задач, решаемых на комплексе:

1) предварительный анализ и детализация волновых сейсмических полей;

2) построение отражающих границ по результатам фазочастотного прослеживания (ФЧП) волн и узкополосной перестраиваемой фильтрации по направлению с использованием данных ГИС;

3) выделение дизъюнктивных нарушений и локальных геологических тел на основе анализа объектно-ориентированных разрезов ФЧП;

4) построение разрезов ФАН-Г по результатам фазовременного анализа и картирование распространения выделенных типов по площади;

5) комплексная интерпретация полученных результатов.

В состав комплекса входят следующие программы (рис. 1):

- MainPrg - программа администрирования процессами вычислений;

- AdmSIDB - программа администрирования базой данных;

- LitoAnalys - программа для исследования и оценки дисперсионных и поглощающих свойств геологических сред;

- PhaseAnalys - программа, реализующая алгоритмы фазочастотного прослеживания волн и фазовременного анализа волнового поля;
- Track Builder - программа узкополосной перестраиваемой фильтрации по направлению;

- Changer - программа преобразования форматов сейсмических данных;

- Contour - программа преобразования внутренних форматов комплекса в форматы системы Golden Software Surfer;

- NewMedium - программа для моделирования волновых сейсмических полей отраженных волн и геологических сред с дисперсией и поглощением.

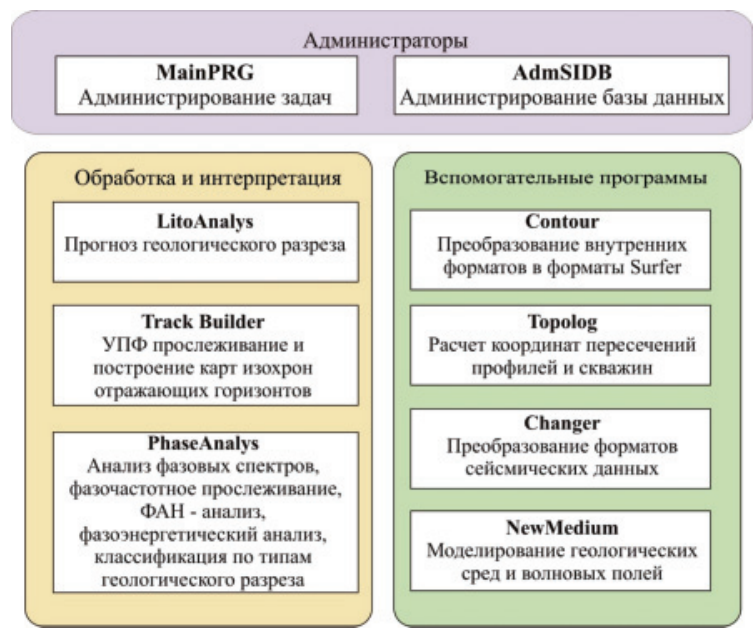

Puc. 1. Состав програм.много комплекса «Геосейф»

Fig. 1. Composition of the software package "Geosef»

Приведенные выше программы поддерживают согласованные между собой форматы входных данных и дают возможность менять граф обработки. Для организации обработки сейсмических профилей и объединения информации в комплекс введена и реализована в формате PARADOX база данных, структурная схема которой приведена на рис. 2.

Администрирование базой данных осуществляется с помощью программы AdmSIDB, а доступ к данным возможен непосредственно из других программ. К важным характеристикам использованной структуры базы данных и схемы взаимодействия программных модулей можно отнести следующие:

- возможность сохранения в базе промежуточных результатов обработки;

- автоматическое взаимное увязывание различных полей всех таблиц базы;

- использование перекрестных ссылок, позволяющее максимально снизить дублирование информации.

Общий подход к решению задач на комплексе «Геосейф» связан с формированием выбранного маршрута в соответствии с принятым графом обработки от имеющихся исходных данных к нужным результатам. Возможности построения маршрутов обеспечиваются как средствами меню, так и путем составления последовательности выполняемых программ. Примеры таких маршрутов представлены на рис. 3-5. 


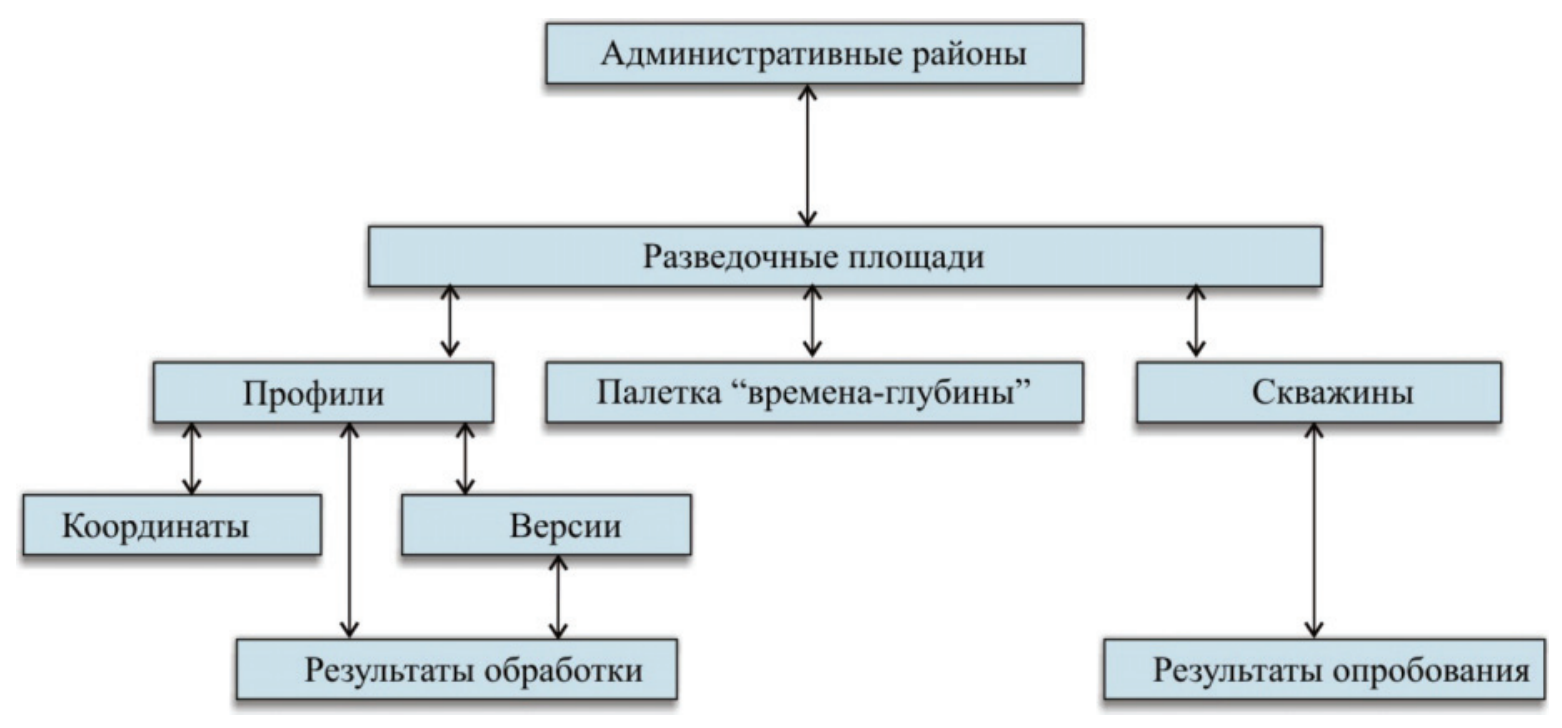

Pис. 2. Структура базы данных комплекса «Геосейф»

Fig. 2. Structure of the database of «Geoseif» complex

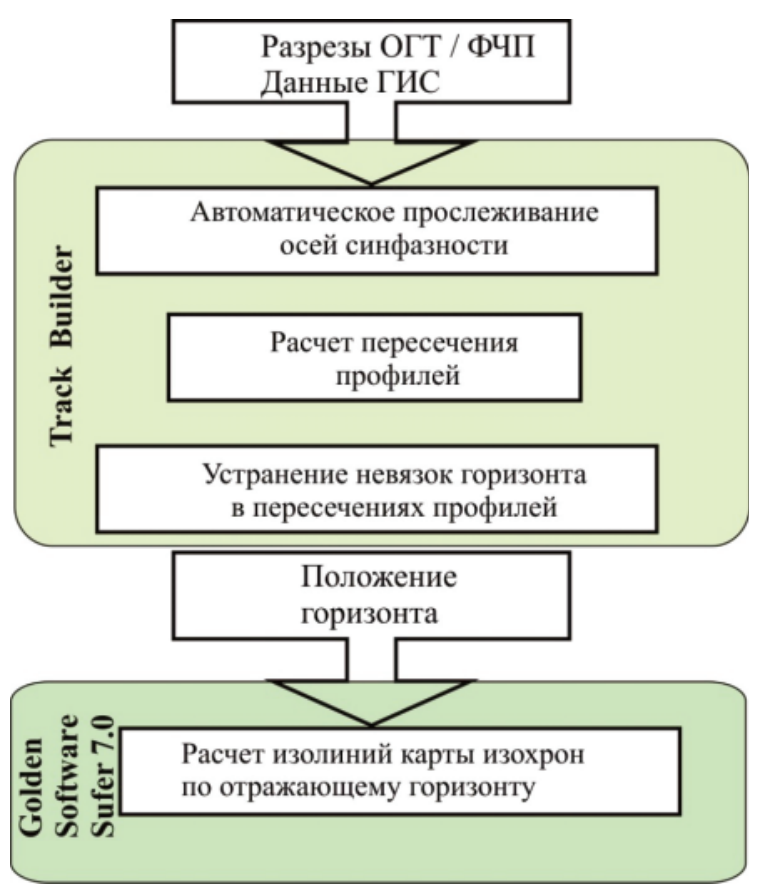

Рис.3. Общал последовательность построения карт изохрон отражающего горизонта

Fig. 3. General sequence of mapping isochrones of the reflecting horizon

Так, например, задача картопостроения решается на начальном этапе с помощью программы Track Builder, в которой на основании данных ГИС, результатов автоматического прослеживания осей синфазности и увязки горизонта в пересечениях профилей решается задача выделения и отождествления целевого отражающего горизонта. Затем средствами программы Track Builder устраняются систематические и линейные невязки положения горизонта в пересечениях профилей. Конечный этап - построение изолиний, вы- полняется с использованием дополнительных программных продуктов (в данном случае - Golden Software Surfer 7.0).

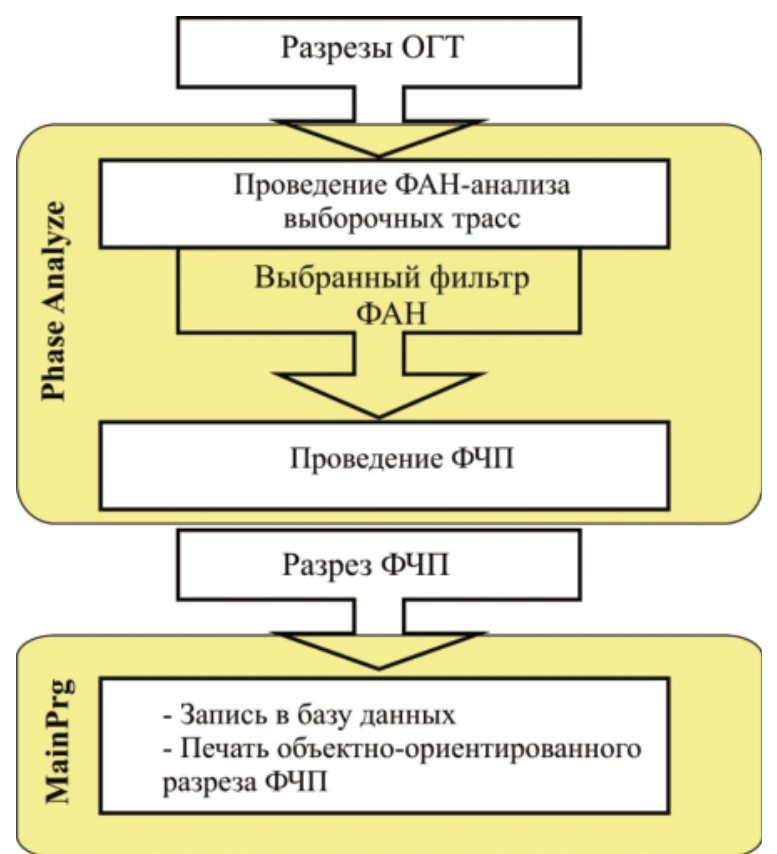

Рис. 4. Последовательность решения задачи прослеживания

Fig. 4. Sequence of solving the tracking problem

При решении задачи прослеживания с неравновесной обработкой на начальном этапе с помощью программы PhaseAnalys выполняется выборочный ФАН-анализ нескольких суммотрасс во временном интервале исследуемых отложений. По результатам изучения ФАН-образов принимается решение о выборе фильтра ФАН, который будет использоваться в качестве весовой функции при выполнении прослеживания. Затем с помощью той же про- 
граммы PhaseAnalys выполняется прослеживание для выбранного временного разреза. Результат прослеживания записывается в базу данных и с помощью программы MainPrg выводится объектноориентированный разрез ФЧП.

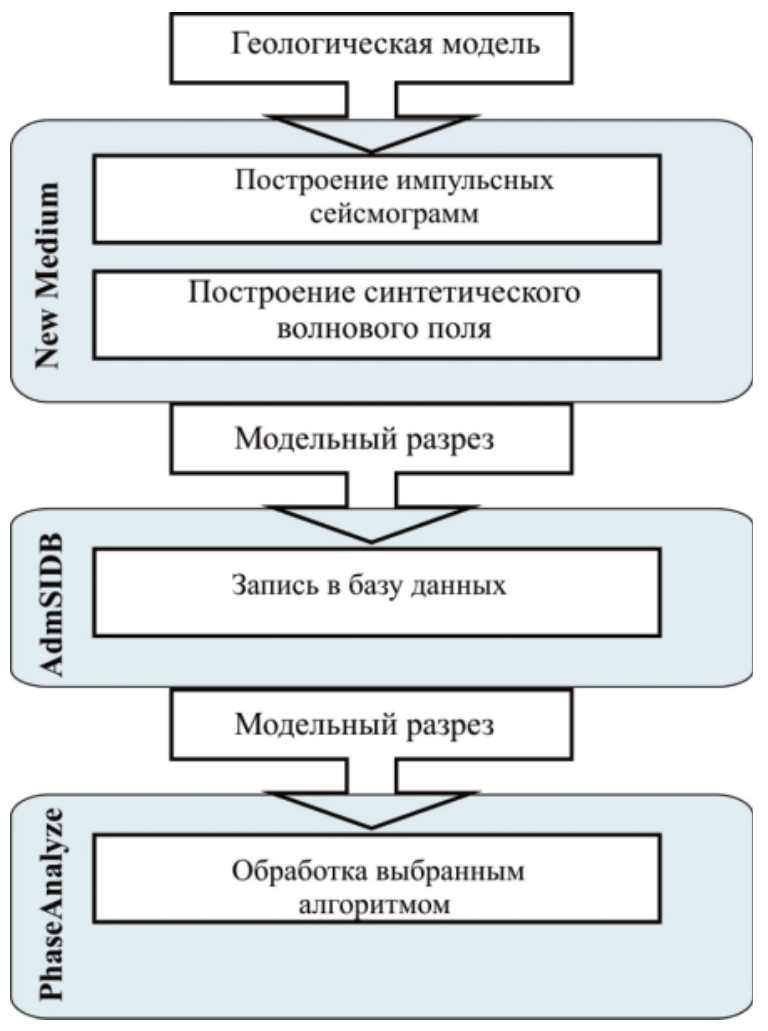

Pис.5. Исследование эффективности работы алгоритмов на модельных волновых полях

Fig. 5. Investigation of the algorithms performance on model wave fields

При исследовании эффективности разрабатываемых алгоритмов на модельных волновых полях, в соответствии с выбранной геологической моделью, с помощью программы NewMedium строится синтетический временной разрез, который записывается в базу данных с помощью программы AdmSIDB. Затем этот разрез обрабатывается по соответствующему алгоритму с помощью программы PhaseAnalys.

\section{Методика детальной обработки временных разрезов ОГТ и ВСП на основе разработанных алгоритмов фазочастотного анализа сейсмических сигналов}

На основе установленных свойств ФЧХ отраженных волн и реализованных в комплексе «Геосейф» фазочастотных алгоритмов анализа сейсмических волновых полей в рамках данной работы была предложена методика и реализована технология решения различных задач прогноза нефтегазоносности по результатам обработки временных разрезов ОГТ с привлечением данных ГИС и результатов испытаний скважин.

В основных чертах эта методика включает следующие этапы:
1. Предварительное изучение, анализ структуры и динамических свойств обрабатываемых временных разрезов ОГТ. В результате выполнения этого этапа устанавливается исследуемый временной интервал, соответствующий выделяемому продуктивному горизонту, определяется полоса частот, в которой сосредоточена основная энергия сейсмических сигналов, распределение волн по кажущимся скоростям, изучаются особенности формы выделяемых отражений, определяются их спектры. Данные, полученные на этом этапе, используются далее для выбора параметров процедур фазочастотного анализа.

2. Проводится фазовременной анализ отдельных суммотрасс для временного интервала, соответствующего анализируемой части разреза. Полученные результаты, с одной стороны, используются для выбора параметров фазочастотного прослеживания, а с другой стороны, применяются для последующих построений разрезов ФАН-Г.

3. Методами фазочастотного прослеживания для целевых толщ выполняется построение объектно-ориентированных фазовых разрезов. С использованием результатов ФАН-анализа и материалов вертикального сейсмического профилирования, акустического каротажа и ГИС на построенных разрезах проводится стратиграфическая привязка отражающих горизонтов, изучение структурных особенностей продуктивных пластов, выделение дизъюнктивных нарушений. Для отражений, приуроченных к границам выделяемых объектов, по результатам прослеживания оцениваются их кинематические параметры, которые могут быть использованы в дальнейшем для проведения прогноза, структурных и палеопостроений.

4. В зонах интенсивной интерференции волн отдельные отражения, приуроченные к границам изучаемых толщ, дополнительно прослеживаются методом узкополосной перестраиваемой фильтрации по направлению (кажущейся скорости). При этом осуществляется восстановление формы отдельных отражений для последующей оценки их динамических характеристик. По результатам прослеживания основных отражающих границ при достаточно плотной сети используемых профилей строятся соответствующие карты изохрон или структурные карты по заданным горизонтам.

5. Строятся ФАН-образы для суммотрасс, отнесенных к положению скважин на профилях. Затем полученные образы объединяются в классы в зависимости от типов геологического разреза, выделенных на основании ГИС, peзультатов бурения и испытания скважин. Это позволяет сформировать набор эталонных ФАН-образов, для которых определяются признаки для последующей автоматической классификации. 
6. Строятся разрезы ФАН-Г, составленные из ФАН-образов суммотрасс, выбранных с заданной дискретностью вдоль временного разреза. При сопоставлении полученных ФАН-образов для изучаемых продуктивных отложений с эталонными на разрезах ФАН-Г выделяются однородные зоны, соответствующие определенному типу геологического разреза. На этом этапе для выделения типов разрезов могут также применяться алгоритмы автоматической классификации. Результаты классификации выносятся на карты.

7. Проводится комплексная интерпретация всех материалов обработки с учетом имеющихся данных ГИС, результатов испытания скважин, структурных построений. На этой основе строится прогноз нефтегазоносности продуктивных отложений на исследуемой площади и даются рекомендации на заложение скважин.

Результаты применения данной методики к обработке материалов сейсмических исследований в пределах Двуреченско-Карандашовской зоны обсуждаются в следующем разделе.

\section{Прогноз типов геологического разреза}

и выделение нефтеперспективных объектов

\section{в Двуреченско-Карандашовской зоне}

На программно-алгоритмическом комплексе «Геосейф» была проведена опытная обработка временных разрезов ОГТ по сети выборочных профилей в пределах Двуреченско-Карандашовской зоны. Целевым объектом исследований являлись продуктивные отложения верхней юры, имеющие тонкослоистую структуру. В связи с этим обработка материалов ОГТ была направлена на достижение максимально возможной разрешенности сейсмической записи с сохранением когерентности регистрируемых отражающих горизонтов. В результате детальной обработки исходных временных разрезов ОГТ по программам комплекса «Геосейф» были получены разрезы фазочастотного прослеживания (ФЧП) и разрезы ФАН-Г фазовременного анализа. Построение разрезов осуществлялось для временного интервала 1,900-2,300 с., т. е. для интервала, в котором регистрируются отражения от границ баженовской, георгиевской, васюганской и тюменской свит, несущие информацию о геологическом строении целевого объекта исследований.

Слабая изученность территории исследований бурением (на исследуемых площадях пробурено всего лишь четыре разведочных скважины), близость к Крапивинскому месторождению и ограниченный объем обработанных профилей определили специфику применения предложенной авторами статьи методики прогноза на территории Двуреченско-Карандашовской зоны. Так, особенностью проведенной типизации в этих зонах явилось то, что она осуществлялась с опорой на результаты, ранее полученные на соседней, достаточно хорошо изученной бурением Крапивинской площади. Ограниченный объем скважинной информа- ции, полученной непосредственно на территории исследований, позволил выделить лишь три типа геологического разреза (I, II, и III). При этом ФАНобразы I типа характеризуют зоны с высокодебитным притоком нефти, а ФАН-образы II и III типов - зоны с невысоким притоком нефти. ФАН-образы IV, V и VI типов геологического разреза (неперспективные с позиций наличия углеводородов) определены путем экстраполяции с соседней Крапивинской площадью.

В результате проведенного ФАН-анализа территория Двуреченско-Карандашовской зоны была районирована по выделенным типам геологического разреза следующим образом (рис. 6). Из рассмотрения карты районирования (рис. 6) видно, что в целом для всей зоны характерна пестрота геологического разреза. Наибольшим развитием пользуется III тип разреза. Наиболее перспективный I тип разреза распространен в зоне весьма ограниченно, занимая приблизительно одну пятую часть Двуреченского л.п. Второй, менее перспективный, тип разреза развит главным образом в пределах Двуреченской площади. Участки с этим типом разреза встречаются как внутри Двуреченского л.п., так и в его периферийных частях. Третий тип разреза, характеризующийся как тип с низкими перспективами, распространен исключительно широко, особенно в Карандашовской части рассматриваемой зоны. IV, V и, особенно, VI типы разреза имеют небольшое распространение и часто приурочены к зонам погружения или переферийным частям поднятий, заходя в редких случаях в пределы самих поднятий.

Проведенный анализ разрезов ФАН-Г показал, что типизация геологического разреза продуктивного интервала принципиально возможна в условиях слабой разбуренности территории. Для эталонирования ФАН-образов по типам разреза в этом случае используется информация единичных скважин, пробуренных непосредственно на территории исследований и привлекаются данные скважин по соседним площадям.

\section{Заключение}

Предлагаемая методика прогнозирования типов геологического разреза (с выделением отдельных объектов) основана на изучении фазочастотных характеристик (ФЧХ) отраженных волн, зафиксированных на временных сейсмических разрезах общей глубинной точки. Высокая эффективность геологической интерпретации с применением этой методики достигается за счет детального расчленения разреза тонкослоистой продуктивной толщи, установления связей значений ФЧХ с зональными и локальными литологическими и тектоническими неоднородностями разреза, выявленными по данным бурения. При этом выявленные корреляционные связи, благодаря сохранению общих закономерностей геологического строения, интерполируются и экстраполируются в межскважинное и заскважинное пространство. Для реали- 


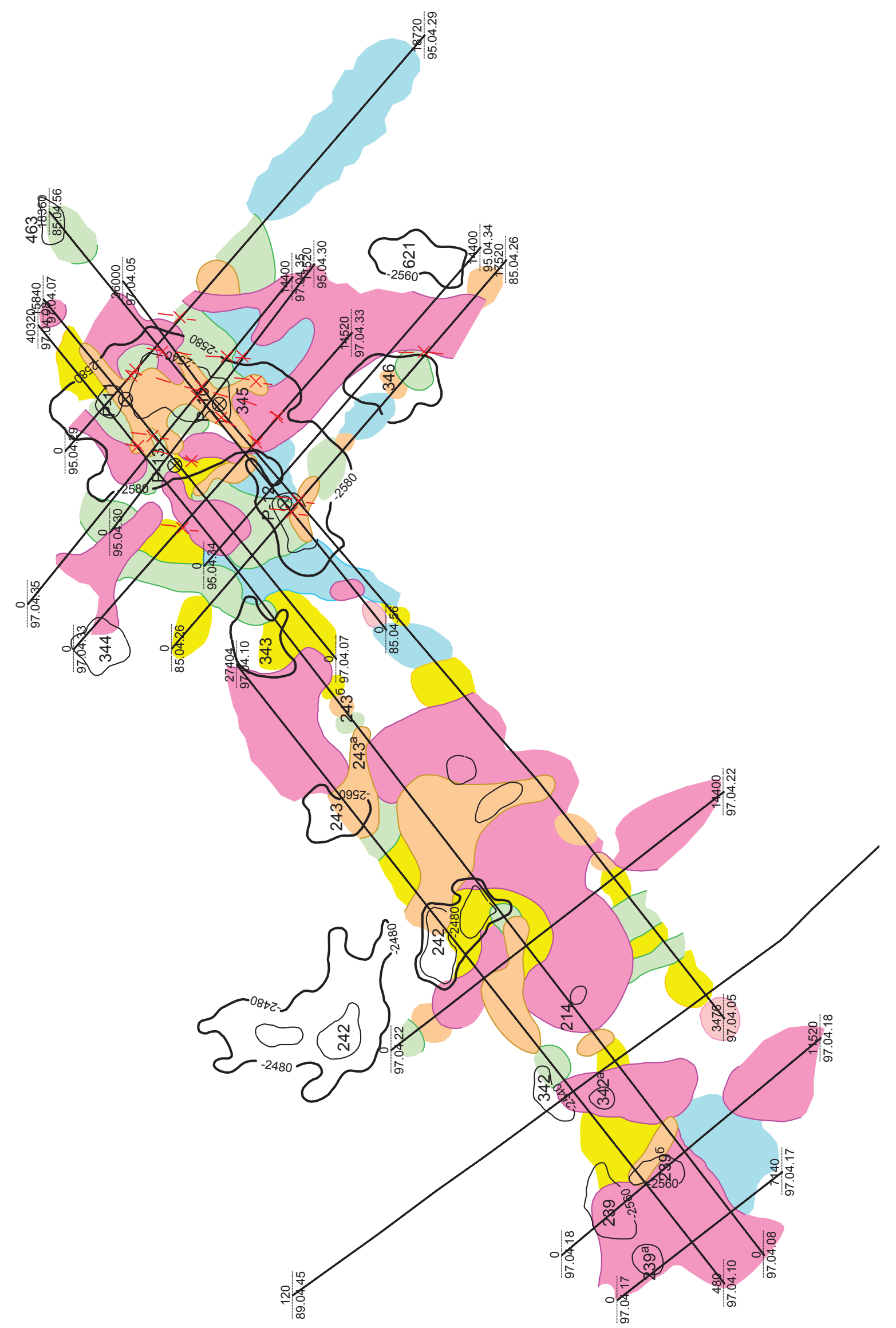




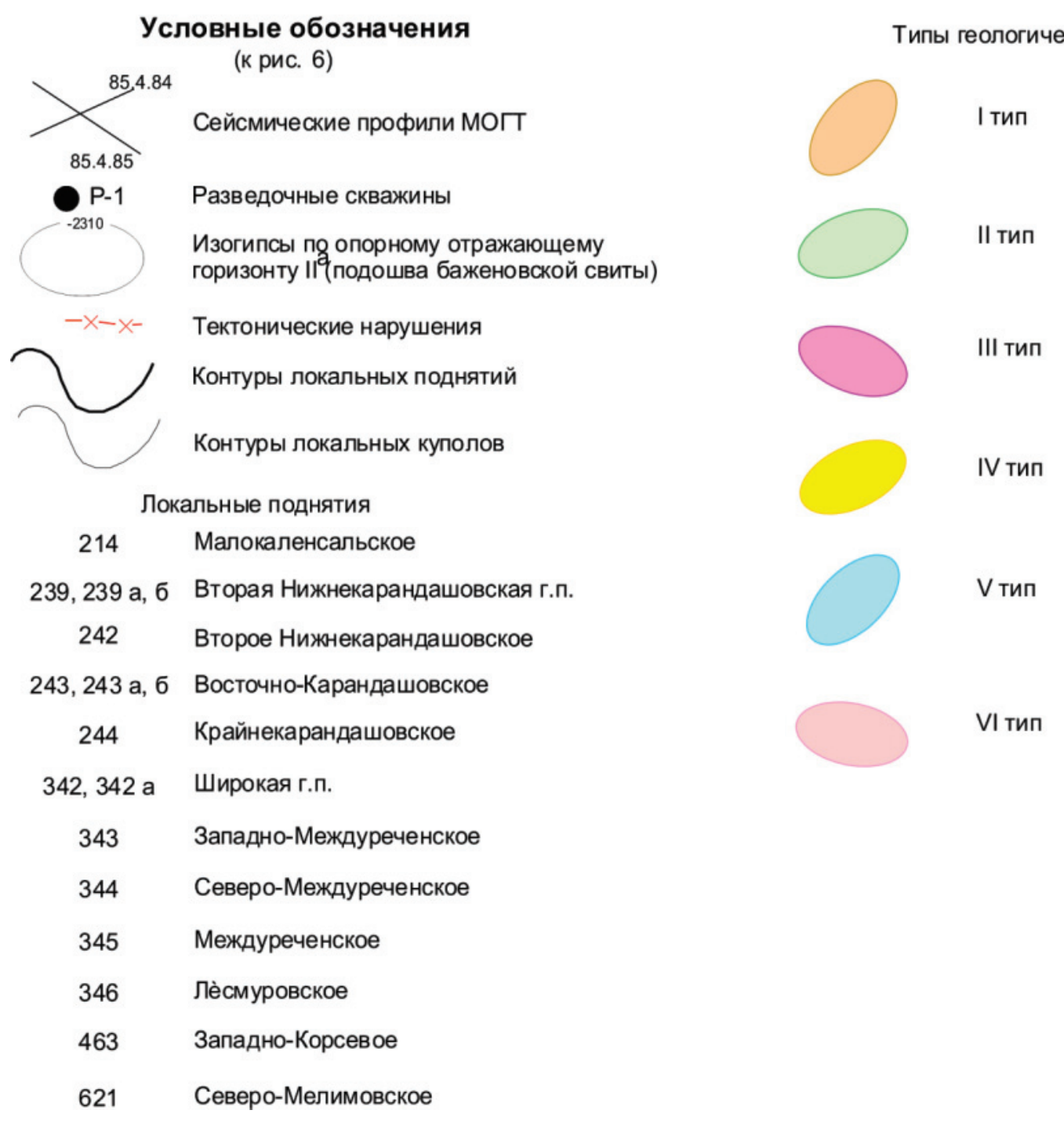

Puс. 6. Карта типов геологического разреза по данным ФАН-Г. Междуреченско-Карандашовская зона

Fig. 6. Map of types of geological section according to FAN-G. Mezhdurechensko-Karandashovskaya zone

зации предложенной методики использован программно-алгоритмический комплекс «Геосейф», позволяющий организовать детальную обработку и интерпретацию сейсмических данных в соответствии с выбранным маршрутом обработки от имеющихся исходных данных к нужным результатам. В результате применения рассмотренной

\section{СПИСОК ЛИТЕРАТУРЫ}

1. Крылов Д.Н. Детальный прогноз геологического разреза в сейсморазведке. - М.: Недра, 2007. - 195 с.

2. Немова В.Д., Бордюг М.А., Ревяко А.В. Сейсмогеологическое моделирование изменений свойств отложений баженовской свиты в межскважинном пространстве // Технологии сейсморазведки. - 2013. - № 2. - С. 72-97.

3. Самаркин М.А. Теоретическое и модельное обоснование методики определения типов геологического разреза переменной толщины в межскважинном пространстве //Журнал Геофизика. - 2003. - Специальный выпуск. - С. 85-89. методики проведен фазовременной анализ территории Двуреченско-Карандашовской зоны и построена карта районирования по выделенным типам геологического разреза.

Работа выполнена при поддержке гранта РФФИ (№ 18-07-01007).

4. Istekova S.A., Umirova G.K. Characteristics of Seismic Data Used To Study the Reservoir of South Torgay Oil and Gas Basin // International Journal of Innovative Research in Science, Engineering and Technology. - 2014. - V. 3. - Iss. 11. P. $17382-17394$.

5. Комплексное спектрально-скоростное прогнозирование типов геологического разреза и фильтрационно-емкостных свойств коллекторов / Е.А. Копилевич, И.А. Мушин, Е.А. Давыдова, М.Л. Афанасьев. - Ижевск: Институт компьютерных исследований, 2010. - 248 с.

6. Иванченков В.П., Кочегуров А.И., Нгуен Суан Хунг. Прогноз коллекторских свойств пород на основе взаимных фазовых 
спектров отраженных сейсмических волн // Нефтяное хозяйство. - 2016. - № 5. - С. 24-28.

7. Новая методика регионального прогноза высокопродуктивных баженовских коллекторов Западной Сибири / Е.А. Копилевич, М.Б. Скворцов, Н.Д. Сурова, Г.В. Кузнецов // Геология нефти и газа. - 2016. - № 4. - С. 58-63.

8. Прогноз типов геологического разреза на основе фазовременного анализа данных сейсмических / В.П. Иванченков, А.И. Кочегуров, Нгуен Суан Хунг, О.В. Орлов // Известия Томского политехнического университета. Инжиниринг георесурсов. - 2017. - Т. 328. - № 4. - С. 55-66.

9. Сильвиа М.Т., Робинсон Э.А. Обратная фильтрация геофизических временных рядов при разведке на нефть и газ. - М.: Недра, 1983. - 382 c.

10. Mohamed Mhmod, Feng Xuan, Xu Cong. Parameters effects on spiking deconvolution of land seismic data // Global Geology. 2015. - V. 18 (4). - P. 226-231.

11. Романов В.В. Возможности повышения разрешенности сейсмограмм метода преломленных волн (МПВ) // Технологии сейсморазведки. - 2013. - № 4. - С. 67-73.

12. Станкевич Л.С. Обработка сейсмических разрезов методом динамического сжатия // Геофизика, геофизическое приборостроение. - 2015. - № 1 (21). - С. 78-85.

13. Митрофанов Г.М. Гомоморфная фильтрация и слепая деконволюция // Технологии сейсморазведки. - 2015. - № 1. C. $46-56$.

14. Egbai J.C., Atakpo E., Aigbogun C.0. Predictive deconvolution in seismic data processing in Atala prospect of rivers State, Nigeria // Advances in Applied Science Research. - 2012. - V. 3 (1). P. 520-529.
15. Lindberg D.V., More H. Blind Categorical Deconvolution in TwoLevel Hidden Markov Models // IEEE Transactions on Geoscience and Remote Sensing. - 2014. - V. 52. - P. 7435-7447.

16. Idan Ram, Israel Cohen. Multichannel Deconvolution of Seismic Signals Using Statistical MCMC Methods // IEEE Transactions on signal processing. - 2010. - V. 58. - № 5. - P. 2757-2770.

17. Deconvolution of Seismic Signals in Time and Frequency Domain / Kedarnath Senapati, Aurobinda Routray, Santosh Dhubia, William Kumar Mohanty // International Journal of Signal Processing, Image Processing and Pattern Recognition processing and Pattern Recognition. - 2011. - V. 4. - № 1. - P. 29-47.

18. Optimal Seismic Deconvolution: Distributed Algorithms / K.N. Plataniotis, S.K. Katsikas, D.G. Lainiotis, A.N. Venetsanopoulos // IEEE Transactions on Geoscience and Remote Sensing. - 1998. - V. 36. - P. 779-792.

19. Veeken P.C.H., Da Silva M. Seismic inversion methods and some of their constraints // First break. - 2004. - V. 22. - P. 47-70.

20. Kazemi N., Bongajum E., Sacchi M. Surface-Consistent Sparse Multichannel Blind Deconvolution of Seismic Signals // IEEE Transactions on geoscience and remote sensing. - 2016. V. 54. - P. 3200-3207.

21. Фазочастотная деконволюция сейсмических волн / А.И. Кочегуров, Е.А. Кочегурова, И.Э. Ильясова, V. Geringer, K. Reif // Известия Томского политехнического университета. Инжиниринг георесурсов. - 2017. - Т. 328. - № 12. - С. 84-93.

Поступила 09.05.2019 2.

\section{Информация об авторах}

Кочегуров А.И., кандидат технических наук, доцент отделения информационных технологий Инженерной школы информационных технологий и робототехники Национального исследовательского Томского политехнического университета.

Иванченков В.П., кандидат технических наук, доцент отделения информационных технологий Инженерной школы информационных технологий и робототехники Национального исследовательского Томского политехнического университета.

Орлов О.В., кандидат технических наук, доцент отделения информационных технологий Инженерной школы информационных технологий и робототехники Национального исследовательского Томского политехнического университета. 
UDC 550.834

\title{
FORECAST OF GEOLOGICAL PROFILE BASED ON DETAILED PROCESSING OF SEISMIC MATERIALS IN THE SOFTWARE-ALGORITHMIC COMPLEX «GEOSEIF»
}

\author{
Alexander I. Kochegurov', \\ kaicc@tpu.ru \\ Viktor P. Ivanchenkov', \\ am@am.tpu.ru \\ Oleg V. Orlov', \\ orloff.oleg@mail.ru
National Research Tomsk Polytechnic University, 30, Lenin Avenue, Tomsk, 634050, Russia.

Relevance. It is well known that the main objectives of forecasting a geological section are to determine the material composition of sedimentation, to select the productive strata and to assess their petroleum potential. These goals are achieved, among other things, on the basis of detailed processing and interpretation of seismic data with reference to the results of well logging. In modern software and algorithmic complexes for processing and interpreting seismic information, the amplitude and energy characteristics of the reflected waves are mainly used as diagnostic signs for prediction of a geological profile. Phase frequency characteristics for this purpose of the forecast are practically not used. All this leads to the fact that when solving the problems of forecasting a geological profile, the percentage of erroneous decisions is quite large and the allocation of false anomalies of dynamic and kinematic parameters of reflections occurs quite often.

The aim of the research is to obtain a forecast of the geological section using new informative parameters extracted from the instantaneous phase spectra of the reflected seismic waves on the basis of the detailed processing of seismic materials in the software and algorithmic complex «Geoseif».

Methods and means of research: digital signal processing and space-time fields, discrete Fourier transform, mathematical modeling methods, computational experiment.

Results. The composition of the software and algorithmic complex "Geoseif» and the list of tasks solved on it are considered in detail. It is shown that the general approach to solving the problems on the "Geoseif» complex is associated with the formation of the selected route in accordance with the accepted processing graph from the available source data to the desired results. The paper presents the structure of the detailed processing of seismic materials during the implementation of phase-frequency deconvolution algorithms for predicting a geological section. As a result of application of the considered methodology, a phase-time analysis of the territory of the Dvurechensko-Karandashovskaya zone was conducted and a zoning map was drawn for the selected types of geological section. At the same time, the identified correlation links between the values of the phase response and the zonal and local lithological and tectonic inhomogeneities of the section are interpolated and extrapolated into the interwell space.

\section{Key words:}

Geological profile forecast, phase-frequency deconvolution, phase-time analysis,

detailed processing, software and algorithmic complex.

The work was funded by the Russian Foundation for Basic Research Grants under grant No. 18-07-01007.

\section{REFERENCES}

1. Krylov D.N. Detalny prognoz geologicheskogo razreza $v$ seysmorazvedke [The detailed forecast of a geological section in seismic exploration]. Moscow, Nedra publ., 2007. 195 p.

2. Nemova V.D., Bordyug M.A., Revyako A.V. Seismic-geological modeling for characterization of the Bazhenovka fm. reservoir in the inter-well space. Technologies of seismic exploration, 2013, vol. 2, pp. 72-97. In Rus.

3. Samarkin M.A. Teoreticheskoe i modelnoe obosnovanie metodiki opredeleniya tipov geologicheskogo razreza peremennoy tolshchiny v mezhskvazhinnom prostranstve [Theoretical and model reasons for a technique of determination of types of a geological section of variable thickness in interborehole space]. Zhurnal geofizi$k a, 2003$, Special Issue, pp. 85-89.

4. Istekova S.A, Umirova G.K. Characteristics of Seismic Data Used to Study the Reservoir of South Torgay Oil and Gas Basin. International Journal of Innovative Research in Science, Engineering and Technology, 2014, vol. 3, Iss. 11, pp. 17382-17394.

5. Kopilevich E.A., Mushin I.A., Davydova E.A., Afanasyev M.L. Kompleksnoe spektralno-skorostnoe prognozirovanie tipov geolo- gicheskogo razreza i filtratsionno-emkostnykh suoystv kollektorov [Complex spectral-speed forecasting of types of geological section and reservoir properties of reservoirs]. Izhevsk, Institut kompyukh issledovaniy Publ., 2010. 248 p.

6. Ivanchenkov V.P., Kochegurov A.I., Nguyen X.H. Prognoz kollectorskikh svoystv porod na osnove vzaimnykh fazovykh spektrov otrazhennykh seysmicheskikh voln [Prediction of reservoir properties of rocks based on mutual phase spectrums of reflected seismic waves]. Neftyanoe khozyaystvo, 2016, no. 5, pp. 24-28.

7. Kopilevich E.A., Skvortsov M.B., Surova N.D., Kuznetsov G.V. Novaya metodika regionalnogo prognoza vysokoproduktivnykh bazhenovskikh kollektorov Zapadnoy Sibiri [New technique of the regional forecast highly productive bazhenovskikh of collectors of Western Siberia]. Geologiya nefti i gaza, 2016, no. 4, pp. 58-63.

8. Ivanchenkov V.P., Kochegurov A.I., Nguyen X.H., Orlov O.V. Predicting geological section types based on the phase-temporal analysis of seismic observations data. Bulletin of the Tomsk Polytechnic University. Geo Assets Engineering, 2017, vol. 328, no. 4, pp. 55-66. In Rus. 
9. Silvia M.T., Robinson E.A. Obratnaya filtratsiya geofizicheskikh bremennykh ryadov pri razvedke na neft i gaz [The return filtration of geophysical temporary ranks at investigation on oil and gas]. Moscow, Nedra Publ., 1983. 382 p.

10. Mohamed Mhmod, Feng Xuan, Xu Cong. Parameters effects on spiking deconvolution of land seismic data. Global Geology, 2015, vol. 18 (4), pp. 226-231.

11. Romanov V.V. Possibilities of increase in resolution of seismographic records of a method of the refracted waves (MPV). Technologies of seismic exploration, 2013, no. 4, pp. 67-73. In Rus.

12. Stankevich L.S. Obrabotka seysmicheskikh razrezov metodom dinamicheskogo szhatiya [Processing seismic cuts by the method of dynamic compression]. Geofizika, geofizicheskoe priborostroenie, 2015, no. 1 (21), pp. 78-85.

13. Mitrofanov G.M. Homomorphic filtering and deconvolution. Technologies of seismic exploration, 2015, no. 1, pp. 46-56. In Rus.

14. Egbai J.C., Atakpo E., Aigbogun C.0. Predictive deconvolution in seismic data processing in Atala prospect of rivers State, Nigeria. Advances in Applied Science Research, 2012, vol. 3 (1), pp. $520-529$.

15. Lindberg D.V., More H. Blind Categorical Deconvolution in TwoLevel Hidden Markov Models. IEEE Transactions on Geoscience and Remote Sensing, 2014, vol. 52, pp. 7435-7447.
16. Idan Ram, Israel Cohen. Multichannel Deconvolution of Seismic Signals Using Statistical MCMC Methods. IEEE Transactions on signal processing, 2010, vol. 58, no. 5, pp. 2757-2770.

17. Kedarnath Senapati, Aurobinda Routray, Santosh Dhubia, William Kumar Mohanty. Deconvolution of Seismic Signals in Time and Frequency Domain. International Journal of Signal Processing, Image Processing and Pattern Recognition processing and Pattern Recognition, 2011, vol. 4, no. 1, pp. 29-47.

18. Plataniotis K.N., Katsikas S.K., Lainiotis D.G., Venetsanopoulos A.N. Optimal Seismic Deconvolution: Distributed Algorithms. IEEE Transactions on Geoscience and Remote Sensing, 1998, vol. 36, pp. 779-792.

19. Veeken P.C.H., Da Silva M. Seismic inversion methods and some of their constraints. First break, 2004, vol. 22, pp. 47-70.

20. Kazemi N., Bongajum E., Sacchi M. Surface-Consistent Sparse Multichannel Blind Deconvolution of Seismic Signals. IEEE Transactions on geoscience and remote sensing, 2016, vol. 54, pp. 3200-3207.

21. Kochegurov A.I., Kochegurova E.A., Ilyasova I.E., Geringer V., Reif K. Phase-Frequency deconvolution of seismic waves. Bulletin of the Tomsk Polytechnic University. Geo Assets Engineering, 2017, vol. 328, no. 12, pp. 84-93. In Rus.

Received: 9 May 2019.

\section{Information about the authors}

Alexander I. Kochegurov, Cand. Sc., associate professor, National Research Tomsk Polytechnic University.

Viktor P. Ivanchenkov, Cand. Sc., associate professor, National Research Tomsk Polytechnic University.

Oleg V. Orlov, Cand. Sc., associate professor, National Research Tomsk Polytechnic University. 